\title{
An extension of conditional nonlinear optimal perturbation approach and its applications
}

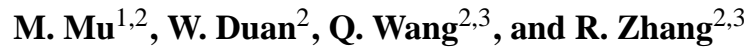 \\ ${ }^{1}$ Key Laboratory of Ocean Circulation and Wave, Institute of Oceanology, Chinese Academy of Sciences, \\ Qingdao 266071, China \\ ${ }^{2}$ LASG, Institute of Atmospheric Physics, Chinese Academy of Sciences, Beijing 100029, China \\ ${ }^{3}$ Graduate University of Chinese Academy of Sciences,Beijing100049, China
}

Received: 21 September 2009 - Revised: 3 March 2010 - Accepted: 9 March 2010 - Published: 13 April 2010

\begin{abstract}
The approach of conditional nonlinear optimal perturbation (CNOP) was previously proposed to find the optimal initial perturbation (CNOP-I) in a given constraint. In this paper, we extend the CNOP approach to search for the optimal combined mode of initial perturbations and model parameter perturbations. This optimal combined mode, also named CNOP, has two special cases: one is CNOP-I that only links with initial perturbations and has the largest nonlinear evolution at a prediction time; while the other is merely related to the parameter perturbations and is called CNOP-P, which causes the largest departure from a given reference state at a prediction time. The CNOP approach allows us to explore not only the first kind of predictability related to initial errors, but also the second kind of predictability associated with model parameter errors, moreover, the predictability problems of the coexistence of initial errors and parameter errors. With the CNOP approach, we study the ENSO predictability by a theoretical ENSO model. The results demonstrate that the prediction errors caused by the CNOP errors are only slightly larger than those yielded by the CNOP-I errors and then the model parameter errors may play a minor role in producing significant uncertainties for ENSO predictions. Thus, it is clear that the CNOP errors and their resultant prediction errors illustrate the combined effect on predictability of initial errors and model parameter errors and can be used to explore the relative importance of initial errors and parameter errors in yielding considerable prediction errors, which helps identify the dominant source of the errors that cause prediction uncertainties. It is finally expected that more realistic models will be adopted to investigate this use of CNOP.
\end{abstract}

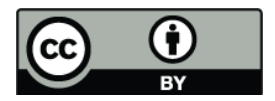

Correspondence to: W. Duan (duanws@lasg.iap.ac.cn)

\section{Introduction}

In 1975, Lorenz classified two kinds of predictability problems (Lorenz, 1975): one is related to initial error with an assumption of a perfect model and referred to as the first kind of predictability; the other is associated with model errors with a perfect initial field and consists of the second kind of predictability. The former has been largely investigated, and many theories and methods have been proposed or introduced (Lorenz, 1965; Toth and Kalnay, 1997; Mu et al., 2003; Mu and Zhang, 2006; Riviere et al., 2008), in which optimal methods are important for estimating the limit of the predictability of weather and climate events. The application of a singular vector (SV; Lorenz, 1965; Farell, 1989) in meteorology is pioneer in this scenario. Considering the limitation of the linear theory of SV, Mu et al. (2003) proposed the approach of conditional nonlinear optimal perturbation (CNOP) to search for the optimal initial perturbation (denoted by "CNOP-I") in a given constraint; its competing aspect is that it considers the effect of nonlinearity. CNOP-I represents the initial error that has the largest negative effect on predictions and has been applied to the predictability studies for ENSO (Duan et al., 2004, 2008, 2009; Duan and Mu, 2006; Mu et al., 2007), the sensitivity analysis for thermohaline circulation (Mu et al., 2004; Sun et al., 2005; Wu and $\mathrm{Mu}, 2009)$, and the adaptive observation for Typhoon (Mu et al., 2009). Riviere et al. (2009) showed an extension of the CNOP approach and used it to estimate the predictability of atmospheric moist processes. Bred vector (Toth and Kalnay, 1997) is another important nonlinear optimal method, which has been used to investigate the first kind of predictability of climate (Cai et al., 2003). All these theories and methods have played an important role in guiding scientists in developing and improving numerical model and even to propose innovative ideas to increase the forecast skill of weather and

Published by Copernicus Publications on behalf of the European Geosciences Union and the American Geophysical Union. 
climate (Houtekamer and Deroma, 1995; Xue et al., 1997; Thompson, 1998; Hamill, 2000; Mu and Zhang, 2006; Mu and Jiang, 2009).

The existing numerical models cannot yet describe exactly the atmospheric and oceanic motions and have model errors, which have caused significant uncertainties in weather and climate predictions (William, 2005; Orrell, 1999, 2003). The effect of model errors on predictability is related to the second kind of predictability (Lorenz, 1975). One important aspect in this field is on the effect of the uncertainties of model parameters on predictability (Lu and Hsieh, 1998; Mu, 2000; Mu et al., 2002). Chu et al. (1999) chose a control parameter and studied the predictability of a numerical model by superimposing different perturbations on this control parameter. Zebiak and Cane (1987), Liu (2002) and Orrell (2003) considered each model parameter and took different values of this parameter to investigate the effect of the uncertainties of the parameters on climate simulation, and to explore the sensitivity of the climate simulation on the parameter perturbations. However, in realistic predictions, the multiple parameters of the model may simultaneously have uncertainties; moreover, there may exist not only model parameter errors but also initial errors. Then how to estimate the predictability limit caused by these combined error modes?

$\mathrm{Mu}$ et al. (2002) considered both initial errors and model parameter errors and classified three predictability problems according to the demands of realistic predictions, the second one of which is to estimate maximum prediction errors caused by both initial errors and parameter errors that respectively satisfy a constraint condition. In a predictability study, it is required to know which combined mode of initial error and parameter error yields the maximum prediction error and what is the patterns of the multiply parameter errors that have the largest effect on predictability. For example, in predictability studies of El Niño-Southern Oscillation (ENSO), it is our desire to know: is it initial error or model error that has a much larger effect on prediction uncertainties? The answer to this question will provide insight on improving ENSO forecast skill (Mu et al., 2007a, b; Duan et al., 2009). By investigating the optimal combined mode of initial errors and model parameter errors and its resultant prediction errors and comparing them with those of the optimal parameter errors, we may answer this question. Thus, we first need to find these optimal modes. Although the above approaches, related to parameter perturbations, are simple in operation, it is very difficult for them to determine these optimal modes. It is, therefore, needed to develop a new approach to study the optimal parameter perturbations and the optimal combined mode of initial perturbation and parameter perturbation, in an attempt to quantify the predictability limit caused by the initial error and model error.

As described above, Mu et al. (2003) have proposed the CNOP approach to find the initial perturbation of the largest evolution at the prediction time, i.e., the aforementioned CNOP-I. CNOP-I is obtained by maximizing the nonlinear evolutions of the initial perturbations in a given constraint (Mu et al., 2003; Mu and Zhang, 2006). Illuminated by the CNOP-I, we attempt to extend the CNOP approach to find the optimal combined mode of initial perturbation and model parameter perturbation and to disclose the optimal parameter perturbations in a given constraint.

The paper is organized as follows. In the next section, the CNOP approach is extended to consist not only of optimal initial perturbation, but also optimal parameter perturbation. The calculation of the extended CNOP is discussed in Sect. 3. In Sect. 4, the CNOP approach is used to illustrate the dominant source of the uncertainties that limit ENSO predictability by a theoretical ENSO model. Finally, the results obtained in this paper are summarized and the physics of CNOPs are discussed in Sect. 5.

\section{Conditional nonlinear optimal perturbation: initial perturbation and parameter perturbation}

We write the evolution equations for the state vector $\boldsymbol{U}$, which may represent surface current, thermocline depth and sea surface temperature, etc., as follows;

$$
\left\{\begin{array}{l}
\frac{\partial U}{\partial t}=F(\boldsymbol{U}, P), \\
\left.\boldsymbol{U}\right|_{t=0}=\boldsymbol{U}_{0},
\end{array} \quad \text { in } \quad \Omega \times[0, \tau]\right.
$$

where $\boldsymbol{U}(x, t)=\left(\boldsymbol{U}_{1}(x, t), \boldsymbol{U}_{2}(x, t), \ldots, \boldsymbol{U}_{n}(x, t)\right)$, and $\boldsymbol{U}_{0}$ is its initial state; $(x, t) \in \Omega \times[0, \tau], \Omega$ is a domain in $R^{n}$, $x=\left(x_{1}, x_{2}, \ldots, x_{n}\right), t=0$ is the initial time and $t=\tau$ with $\tau<+\infty$ is a future time; furthermore, $P=\left(P_{1}, P_{2}, \ldots, P_{\mathrm{m}}\right)$ is model parameters and $P_{i}$ represents one model parameter that is independent of time $t . F$ is a nonlinear differential operator. Assume that the dynamical system equations Eq. (2.1) and the initial state are known exactly, the future state can be determined by integrating Eq. (2.1). The solution to Eq. (2.1) for the state vector $U$ at time $\tau$ is given by

$\boldsymbol{U}(\tau)=M_{\tau}(\boldsymbol{P})\left(\boldsymbol{U}_{0}\right)$.

Here $M_{\tau}(\boldsymbol{P})$ is the propagator of Eq. (2.1) with the parameter vector $\boldsymbol{P}$ and, as described by (2.2), "propagates" the initial value $\boldsymbol{U}_{0}$ to the time $\tau$ in the future.

Considering further the solution $\boldsymbol{U}(\tau)+u(\tau)$ of the Eq. (2.1) with initial value $\boldsymbol{U}_{0}+u_{0}$, we have

$\boldsymbol{U}(\tau)+u(\tau)=M_{\tau}(\boldsymbol{P})\left(\boldsymbol{U}_{0}+u_{0}\right)$,

where $u_{0}$ is the initial perturbation of a time-dependent state $\boldsymbol{U}(t)$ (hereafter as reference state), and $u(\tau)$ describes the nonlinear evolution of this initial perturbation. To facilitate the discussion, we use the denotation $u\left(u_{0} ; \tau\right)$ instead of the $u(\tau)$. 
Assume that a parameter perturbation $p^{\prime}$ is superimposed on a reference parameter $P$, then

$\boldsymbol{U}(\tau)+u\left(p^{\prime} ; \tau\right)=M_{\tau}\left(P+p^{\prime}\right)\left(\boldsymbol{U}_{0}\right)$

where $u\left(p^{\prime} ; \tau\right)$ describes the departure from the reference state $\boldsymbol{U}(\tau)$ (see Eq. 2.2) caused by $p^{\prime}$.

Now we explore the situation that there exists both initial perturbation and parameter perturbation in Eq.(2.2). Then we have

$\boldsymbol{U}(\tau)+u\left(u_{0}, p^{\prime} ; \tau\right)=M_{\tau}\left(P+p^{\prime}\right)\left(U_{0}+u_{0}\right)$.

$u\left(u_{0}, p^{\prime} ; \tau\right)$ is the departure from the reference state $\boldsymbol{U}(\tau)$ caused by the combined error mode $\left(u_{0}, p^{\prime}\right)$.

A nonlinear optimization problem is defined as follows.

$$
J\left(u_{0} ; p^{\prime}\right)=\left\|M_{\tau}\left(P+p^{\prime}\right)\left(\boldsymbol{U}_{0}+u_{0}\right)-M_{\tau}(P)\left(\boldsymbol{U}_{0}\right)\right\|,
$$

and

$$
J\left(u_{0 \delta} ; p_{\sigma}^{\prime}\right)=\max _{u_{0} \in C_{\delta}, p^{\prime} \in C_{\sigma}} J\left(u_{0} ; p^{\prime}\right)
$$

Here $u_{0} \in C_{\delta}$ and $p^{\prime} \in C_{\sigma}$, respectively, are the constraint conditions of the initial perturbations and parameter perturbations, where $C_{\delta}$ and $C_{\sigma}$ are closed and $\delta$ and $\sigma$ distinguish the constraints of initial perturbations and parameter perturbations. The constraint conditions here can simply be expressed as belonging to a ball with a chosen norm; obviously, we can also investigate the situation that the perturbations satisfy some physical laws or other. In addition, we can consider the parameters $p$ in the Eq. (2.1) to be time-dependent and to establish the optimization problems similar to the optimization problem (2.4). Nevertheless, the corresponding computation could be more difficult, which will not be discussed here.

Obviously, the optimization problem (2.4) is a constrained maximization problem. By this optimization problem, one can obtain the optimal combined mode of initial perturbation and parameter perturbation, $\left(u_{0 \delta} ; p_{\sigma}\right)$, which, for the given constraint, induces the largest departure from the reference state $U(t)$ at time $\tau$. It is noticed that, when we consider only initial perturbation or assume that the constraint of the parameter perturbation is $p^{\prime}=0$, the optimization problem (2.4) becomes

$J_{u_{0}}\left(u_{0 \delta}^{I}\right)=\max _{u_{0} \in C_{\delta}}\left\|M_{\tau}(P)\left(\boldsymbol{U}_{0}+u_{0}\right)-M_{\tau}(P)\left(\boldsymbol{U}_{0}\right)\right\|$,

and the initial perturbation $u_{0 \delta}^{I}$ satisfying (2.5) just is the CNOP-I, i.e., the CNOP defined by Mu et al. (2003). Thus, it is clear that the optimal combined mode in (2.4) is an extension of the CNOP proposed in Mu et al. (2003). For convenience, we still named this extended CNOP as CNOP. That is to say, the CNOP hereafter consists of the optimal combined mode of initial perturbation and parameter perturbation; and the aforementioned CNOP-I is a special case of the
CNOP. It has been shown that CNOP-I is a natural generalization of linear singular vector (LSV) to nonlinear regime (Mu et al., 2003), which has been applied in stability, sensitivity, and predictability studies (Mu et al., 2007b; Terwisscha van Scheltinga and Dijkstra, 2008; $\mathrm{Wu}$ and $\mathrm{Mu}, 2009$ ). All these applications demonstrate that CNOP-I is more applicable than LSV in estimating predictability limit.

Another special case of the CNOP is on model parameter perturbation. In fact, when only investigating the effect of model parameter perturbation on a given reference state, it requires one to explore the departure from the reference state at the prediction time. In this case, we can derive from (2.4) the parameter perturbations that cause the largest departure from the given reference state. In (2.4), we neglect the initial perturbation, namely, take the constraint of initial perturbation as $u_{0}=0$, and obtain

$J_{p}\left(p_{\sigma}^{p}\right)=\max _{p^{\prime} \in C_{\sigma}}\left\|M_{\tau}\left(P+p^{\prime}\right)\left(\boldsymbol{U}_{0}\right)-M_{\tau}(P)\left(\boldsymbol{U}_{0}\right)\right\|$,

in which $p_{\sigma}^{p}$ is just such an optimal parameter perturbation in the given constraint. Following the CNOP-I, we call such an optimal parameter perturbation as "CNOP-P".

Although Lu and Hsieh (1998), Fan and Chou (1999), Grimstad et al. (2003) and Aanonsen (2005) used the nonlinear optimization method to explore the model parameters, they devoted their time to solving a minimization problem and to determining the values of the unknown parameter in numerical models. In a predictability study, it is also required to investigate the parameter uncertainties that have the largest effect on prediction results, but this is related to a maximization problem. In essence, this is different from those in the aforementioned works on parameters and cannot be realized by their approaches. The CNOP-P can be used to address this question. In fact, if the $\boldsymbol{U}_{0}$ in (2.3) is regarded as an initial observation, the CNOP not only illustrates the optimal combined mode of initial observational error and model parameter error, but also presents an estimation of the upper-bound maximum prediction errors through its resultant prediction error (see Mu et al., 2002). Correspondingly, the CNOP$\mathrm{P}$ (CNOP-I) error shows an estimation of the upper-bound maximum prediction errors in a perfect initial condition scenario (perfect model scenario) and represents the parameter errors (the initial observational errors) that has the largest effect on predictability. These upper bounds of the maximum prediction errors can be attained. It is the CNOP, CNOP-I and CNOP-P errors that cause the maximum prediction error in their respective scenario. To facilitate the discussion, we hereafter use the term "initial error", rather than "initial observational error", to describe the results obtained in this paper.

In order to employ the CNOP approach in predictability studies, CNOP-I or CNOP-P or CNOP should first be obtained. However, it is very difficult to solve them analytically. One should attempt to compute them numerically. In the next section, we will discuss this question. 


\section{Computations of CNOP, CNOP-I and CNOP-P}

CNOP-I has been computed in many studies. In calculating CNOP-I, some optimization solvers are useful (Powell et al., 1982; Liu and Nocedal, 1989; Birgin et al., 2000). They usually search for CNOP-I along the steepest descent in the direction of the gradient of the corresponding objective function. It is obvious that the gradient is important for capturing the maximal value of the objective function. In large-scale optimization, the gradient of the objective function, with respect to initial perturbations, is often obtained by the adjoint method (Le Dimet and Talagrand, 1986). In fact, CNOP and CNOP-P can also be obtained by optimization solvers with a gradient; moreover, the gradient of the objective function, with respect to initial perturbations and parameter perturbations, can be transferred to a particular case of the objective function with respect to augmented initial perturbations. Then the computation of such a gradient is an elementary application of the general adjoint method. Although it is now very well known in meteorology and oceanography (see Lu and Hsieh, 1998), we, for the readers' convenience, still describe here how to compute CNOP and CNOP-P with a gradient.

The existing optimization solvers are often used to compute minimization problems; while CNOPs is related to a constrained maximization problem. In calculating CNOPs, we turn the maximization problem into a minimization problem. In particular, we rewrite the objective function in (2.3) as follows:

$J_{1}\left(u_{0} ; p^{\prime}\right)=-\frac{1}{2}\left[J\left(u_{0} ; p^{\prime}\right)\right]^{2}=$

$-\frac{1}{2}\left\|M_{\tau}\left(P+p^{\prime}\right)\left(\boldsymbol{U}_{0}+u_{0}\right)-M_{\tau}(P)\left(\boldsymbol{U}_{0}\right)\right\|^{2}=$

$-\frac{1}{2}<u\left(u_{0}, p^{\prime} ; \tau\right), u\left(u_{0}, p^{\prime} ; \tau\right)>$,

where $<\cdot\rangle$ is the inner product. Then the maximization problem (2.4) becomes a minimization one. By computing the minimum of the function $J_{1}\left(u_{0} ; p^{\prime}\right)$, the CNOPs can be obtained.

The first-order variational of $J_{1}\left(u_{0} ; p^{\prime}\right)$ is as follows:

$-\delta J_{1}=<u(\tau), \delta u(\tau)>=<\frac{-\partial J_{1}}{\partial u_{0}}, \delta u_{0}>+<\frac{-\partial J_{1}}{\partial p^{\prime}}, \delta p^{\prime}>$,

where, to facilitate the description, we simply use $u$ instead of $u\left(u_{0}, p^{\prime} ; \tau\right)$. Furthermore, $\delta u(t)$ and $\delta p$ can be governed by the following tangent linear model

$$
\left\{\begin{array}{l}
\frac{\partial \delta \boldsymbol{u}}{\partial t}=\frac{\partial F\left(U(t)+u(t) ; P+p^{\prime}\right)}{\partial u} \delta u+\frac{\partial F\left(U(t)+u(t) ; P+p^{\prime}\right)}{\partial p^{\prime}} \delta p \\
\frac{\partial \delta p}{\partial t}=0 \\
\left.\delta \boldsymbol{u}\right|_{t=0}=\delta \boldsymbol{u}_{0}, \\
\left.\delta p\right|_{t=0}=\delta p^{\prime} .
\end{array}\right.
$$

By introducing two Lagrangian Multipliers $\lambda_{1}$ and $\lambda_{2}$, we obtain that

$$
\begin{aligned}
& -\delta J_{1}=<u(\tau), \delta u(\tau)>-\int_{0}^{\tau}<\lambda_{1}(t), \frac{\partial \delta u}{\partial t}- \\
& \frac{\partial F\left(U+u ; P+p^{\prime}\right)}{\partial u} \delta u-\frac{\partial F\left(U+u ; P+p^{\prime}\right)}{\partial p^{\prime}} \delta p>d t- \\
& \int_{0}^{\tau}<\lambda_{2}(t), \frac{\partial \delta p}{\partial t}>d t .
\end{aligned}
$$

With an integration by parts, we can get

$$
\begin{aligned}
& \int_{0}^{\tau}<\lambda_{1}(t), \frac{\partial \delta u}{\partial t}>d t=\int_{0}^{\tau} \frac{\partial}{\partial t}<\lambda_{1}(t), \delta u>d t- \\
& \int_{0}^{\tau}<\frac{\partial \lambda_{1}(t)}{\partial t}, \delta u>d t=<\lambda_{1}(\tau), \delta u(\tau)>- \\
& <\lambda_{1}(0), \delta u(0)>-\int_{0}^{\tau}<\frac{\partial \lambda_{1}(t)}{\partial t}, \delta u>d t
\end{aligned}
$$

and

$$
\begin{aligned}
& \int_{0}^{\tau}<\lambda_{2}(t), \frac{\partial \delta p}{\partial t}>d t=\int_{0}^{\tau} \frac{\partial}{\partial t}<\lambda_{2}(t), \delta p>d t- \\
& \int_{0}^{\tau}<\frac{\partial \lambda_{2}(t)}{\partial t}, \delta p>d t=<\lambda_{2}(\tau), \delta p(\tau)>- \\
& <\lambda_{2}(0), \delta p(0)>-\int_{0}^{\tau}<\frac{\partial \lambda_{2}(t)}{\partial t}, \delta p>d t= \\
& <\lambda_{2}(\tau), \delta p^{\prime}>-<\lambda_{2}(0), \delta p^{\prime}>-\int_{0}^{\tau}<\frac{\partial \lambda_{2}(t)}{\partial t}, \delta p>d t .
\end{aligned}
$$

Then we derive $\delta J$ as follows:

$$
\begin{aligned}
- & \delta J_{1} \\
& =\int_{0}^{\tau}<\frac{\partial \lambda_{1}}{\partial t}, \delta u>d t+<u(\tau)-\lambda_{1}(\tau), \delta u(\tau)>+<\lambda_{1}(0), \delta u_{0}> \\
& +\int_{0}^{\tau}<\lambda_{1}(t),\left[\frac{\partial F\left(U(t)+u(t) ; P+p^{\prime}\right)}{\partial u}\right] \delta u>d t \\
& +\int_{0}^{\tau}<\lambda_{1}(t),\left[\frac{\partial F\left(U(t)+u(t) ; P+p^{\prime}\right)}{\partial p^{\prime}}\right] \delta p>d t \\
& +\int_{0}^{\tau}<\frac{\partial \lambda_{2}(t)}{\partial t}, \delta p>d t+<0-\lambda_{2}(\tau), \delta p^{\prime}>+<\lambda_{2}(0), \delta p^{\prime}> \\
& =\int_{0}^{\tau}<\frac{\partial \lambda_{1}}{\partial t}, \delta u>d t+<u(\tau)-\lambda_{1}(\tau), \delta u(\tau)>+<\lambda_{1}(0), \delta u_{0}>
\end{aligned}
$$




$$
\begin{aligned}
& +\int_{0}^{\tau}<\left[\frac{\partial F\left(U(t)+u(t) ; P+p^{\prime}\right)}{\partial u}\right]^{*} \lambda_{1}(t), \delta u>d t \\
& +\int_{0}^{\tau}<\left[\frac{\partial F\left(U(t)+u(t) ; P+p^{\prime}\right)}{\partial p^{\prime}}\right]^{*} \lambda_{1}(t), \delta p>d t \\
& +\int_{0}^{\tau}<\frac{\partial \lambda_{2}(t)}{\partial t}, \delta p>d t+<0-\lambda_{2}(\tau), \delta p^{\prime}>+<\lambda_{2}(0), \delta p^{\prime}> \\
& =\int_{0}^{\tau}<\frac{\partial \lambda_{1}}{\partial t}+\left[\frac{\partial F\left(U(t)+u(t) ; P+p^{\prime}\right)}{\partial u}\right]^{*} \lambda_{1}(t), \delta u>d t \\
& +<u(\tau)-\lambda_{1}(\tau), \delta u(\tau)>+<\lambda_{1}(0), \delta u_{0}> \\
& +\int_{0}^{\tau}<\frac{\partial \lambda_{2}}{\partial t}+\left[\frac{\partial F\left(U(t)+u(t) ; P+p^{\prime}\right)}{\partial p^{\prime}}\right]^{*} \lambda_{1}(t), \delta p>d t \\
& +<0-\lambda_{2}(\tau), \delta p^{\prime}>+<\lambda_{2}(0), \delta p^{\prime}>
\end{aligned}
$$

where the sign " $[\cdot]^{*}$ " denotes an adjoint operator. Therefore, by (3.2) and (3.5), we have

$$
\frac{\partial J_{1}}{\partial u_{0}}=-\lambda_{1}(0)
$$

and

$$
\frac{\partial J_{1}}{\partial p^{\prime}}=-\lambda_{2}(0)=-\int_{0}^{\tau}\left[\frac{\partial F\left(U(t)+u(t) ; P+p^{\prime}\right)}{\partial p^{\prime}}\right]^{*} \lambda_{1}(t) d t
$$

where $\lambda_{1}(t)$ and $\lambda_{2}(t)$ satisfies

$$
\left\{\begin{array}{l}
\frac{\partial \lambda_{1}}{\partial t}+\left[\frac{\partial F\left(U(t)+u(t) ; P+p^{\prime}\right)}{\partial u}\right]^{*} \lambda_{1}=0, \\
\frac{\partial \lambda_{2}}{\partial t}+\left[\frac{\partial F\left(U(t)+u(t) ; P+p^{\prime}\right)}{\partial p^{\prime}}\right]^{*} \lambda_{1}=0, \\
\left.\lambda_{1}\right|_{t=\tau}=u(\tau), \\
\left.\lambda_{2}\right|_{t=\tau}=0 .
\end{array}\right.
$$

The Eq. (3.8) is the adjoint equation of the Eq. (3.3). By integrating the Eq. (3.8), we can obtain the gradient $\partial J_{1} / \partial u_{0}$ and $\partial J_{1} / \partial p^{\prime}$. With this gradient information, the CNOPI, CNOP-P and CNOP can be computed by optimization solvers such as Spectral Projected Gradient 2 (SPG2; Birgin et al., 2000), Sequential Quadratic Programming (SQP; Powell et al., 1982) and Limited memory Broyden-FletcherGoldfarb-Shanno for bound-constrained optimization (LBFGS-B; Liu and Nocedal, 1989; Zhu et al., 1997).

It is easily seen that the Eq. (3.8) is established on the Eq. (3.9). And the Eq. (3.9) is the adjoint of the tangent linear model of Eq. (2.1) and can be used to calculate the gradient $\partial J_{1} / \partial u_{0}$ related to CNOP-I.

$$
\left\{\begin{array}{l}
\frac{\partial \lambda_{1}}{\partial t}+\left[\frac{\partial F\left(U(t)+u(t) ; P+p^{\prime}\right)}{\partial u}\right]^{*} \lambda_{1}=0, \\
\left.\lambda_{1}\right|_{t=\tau}=u(\tau),
\end{array}\right.
$$

This suggests that, if there are adjoint models Eq. (3.9) related to initial perturbations, one can easily modify them as the adjoint models Eq. (3.8) to calculate CNOPs.

In the above, we derive the gradient of the objective function $J_{1}$, with respect to initial perturbations and parameter perturbations, by introducing two Lagrangian Multipliers $\lambda_{1}$ and $\lambda_{2}$ for an augmented initial perturbation equation. The approach could clearly present the relationship between the adjoint model with the initial perturbations and the model with parameter perturbations. Of course, this gradient can also be obtained by the usual differentiation of the objective function, with respect to initial condition and parameters (i.e. using the definition of the derivative of a nonlinear operator) (see Shutyaev et al., 2008). In addition, since CNOP is related to a constrained optimization problem, the constraint condition itself is also enforced in optimization. In different optimization algorithms, the constraints, together with the objective functions, may show different manners to determine the new iteration points in the optimization processes (see Powell et al., 1982; Birgin et al., 2000). In the L-BFGS-B solver to be adopted in the next section, the constraint defines a feasible region of the initial perturbations $u_{0}$ and the feasible direction of the steepest decent is obtained by the gradient projection method (Rosen, 1960). Given that $u_{0}$ is in the feasible region, the gradient $\nabla J_{1}$ is computed. The direct gradient decent gives $u_{0}-\mu \nabla J_{1}$ ( $\mu$ is a step size). But this vector may no longer be in the feasible region. To keep this feasibility, the new iteration points are obtained by projecting $u_{0}-\mu \nabla J_{1}$ onto the feasible region defined by the constraint. By this, the updated iteration points still lie in the feasible region, which finally makes the extreme points correspond to minimal value satisfy the constraint condition as well. This is only a rough description. For more details, the reader is referred to Zhu et al. (1997).

\section{Application of CNOPs to a simple coupled ocean-atmosphere model for ENSO}

The ENSO, a prominent climate phenomenon in the coupled ocean-atmosphere system of the tropical Pacific, has a great impact on the global climate. While significant progress has been made in ENSO theories and predictions over the years (see the review of Wang and Picaut, 2004), there still exists considerable uncertainties in realistic ENSO predictions (Jin et al., 2008; Tang et al., 2008). Many studies explored ENSO predictability from the view of initial error growth (Moore and Kleeman, 1996; Samelson and Tziperman, 2001) and showed that initial error may have a large effect on ENSO predictions. It is well known that, in realistic predictions of ENSO, the prediction uncertainties are generally caused by initial errors and model errors. Furthermore, an increasing number of studies have indicated that the errors which existed in some model parameters influence the ENSO forecast skill at a particular time scale (Liu, 2002; Wu et al., 1993; 
Zebiak and Cane, 1987). Then the question is, in initial errors and model parameter errors; which one plays the major role in yielding considerable uncertainties of ENSO predictions? In this section, we will try to address this by using the CNOP approach.

\subsection{The coupled ENSO model}

The model we used in this paper is the theoretical coupled ocean-atmosphere model of Wang and Fang (1996) (hereafter referred to as WF96), which has been used to investigate the predictability of ENSO by Duan et al. $(2004,2008)$ and Duan and Mu (2006). This model consists of two dimensionless equations: one describing the evolutions of the anomalous SST $T$ in the equatorial eastern Pacific, and the other depicting those of the anomalous thermocline depth $h$.

$\left\{\begin{array}{l}\frac{d T}{d t}=a_{1} T-a_{2} h+\sqrt{\frac{2}{3}} T\left(T-a_{3} h\right), \\ \frac{d h}{d t}=b(2 h-T),\end{array}\right.$

where

$a_{1}=\bar{T}_{z}^{\prime}+\bar{T}_{x}^{\prime}-\alpha_{\mathrm{s}}^{\prime}$,

$a_{2}=\left(\mu+\delta_{1}\right) \bar{T}_{x}^{\prime}$,

$a_{3}=\mu+\delta_{1}$

$b=\frac{2 \alpha}{p\left(1-3 \alpha^{2}\right)}$.

The coefficients $a_{1}$ and $a_{2}$ involve basic state parameters $\bar{T}_{x}^{\prime}$ and $\bar{T}_{z}^{\prime}$, which characterise, respectively, the mean temperature difference between the eastern and western basins and between the surface and subsurface water, and reflect the climatological annual cycle of the basic state. $\delta_{1}$ represents the contribution of the horizontal temperature advection by anomalous zonal currents to local SST variation. Two essential coupling parameters (nondimensional) are presented in this model. One is the air-sea coupling coefficient, $\alpha=$ $\left(\frac{L_{0}}{L_{y}}\right)^{2}$, where $L_{0}$ is the oceanic Rossby radius of deformation and $L_{y}$ is the characteristic meridional length scale of the coupled ENSO mode. Another coupling parameter is the thermocline effect coefficient $\mu=\frac{\mu_{*} H_{1}}{\theta}$, which measures the degree of coupling between thermocline fluctuation and SST. These two parameters are often empirically determined in the model and have uncertainties. In this paper, we choose $\alpha=0.0212$ and $\mu=1.525$ as the given values of the two parameters. The nondimensional parameter $b$ is a function of $\alpha$ and the model parameters $p=\left(1-H_{1} / H\right)\left(L_{0} / L_{\mathrm{s}}\right)^{2}$, where $H$ and $H_{1}$, respectively, are the mean depth of the thermocline and the mixed layer and $L_{\mathrm{s}}$ is the Ekman spreading length. For the values of these parameters, the reader is referred to Table 1 in WF96.
The steady solution $O(0,0)$ represents the climatological annual cycle, in which both SST and the depth of thermocline are normal. In this paper, the model is integrated by the fourth-order Runge-Kutta scheme with $d t=0.01$, which represents one day.

\subsection{Estimation of prediction errors for El Niño events by CNOP, CNOP-I, and CNOP-P}

With an initial constraint $U_{0} \in\left\{U_{0} \mid\left\|U_{0}\right\| \leq \rho_{0}\right\}$, where $\left\|U_{0}\right\|=\max \left\{\left|T_{0}\right|,\left|h_{0}\right|\right\}$, Duan et al. (2009) studied the CNOP-I superimposed on the climatological annual cycle in the WF96 model and demonstrated that the CNOP-I anomalies $\left(T_{0}, h_{0}\right)=\left(-\rho_{0}, \rho_{0}\right)$ with different positive values of $\rho_{0}$ evolve into different intensities of El Niño events. These initial anomalies have the robust patterns of negative SST and positive thermocline depth anomalies, which agree with the observations qualitatively (Duan et al., 2004), and act as the optimal precursors of El Niño events. In this paper, we choose $\rho_{0}=0.05$ and 0.08 , then obtain two initial precursory anomalies $\left(T_{0}, h_{0}\right)=(-0.05,0.05)$ and $(-0.08,0.08)$, which are dimensionless and represents dimensional $\left(-0.1^{\circ} \mathrm{C}, 2.5 \mathrm{~m}\right)$ and $\left(-0.16^{\circ} \mathrm{C}, 4.0 \mathrm{~m}\right)$ of SSTA and the thermocline depth anomaly in the equatorial eastern Pacific. We consider these initial precursors occurring in January, April, July, and October, respectively. And for each initial precursor time, the above two initial anomalies induce two different intensities of El Niño events. The initial anomaly $(-0.05,0.05)$ develops a weak El Niño event, while $(-0.08,0.08)$ evolves into a relatively strong El Niño event (see Mu and Duan, 2003; Duan et al., 2009). For convenience, we denote the weak (strong) El Niño events with initial precursor time January, April, July and October as $U_{\mathrm{Jan}}^{\mathrm{W}}\left(U_{\mathrm{Jan}}^{S}\right), U_{\mathrm{Apr}}^{\mathrm{W}}\left(U_{\mathrm{Apr}}^{S}\right), U_{\mathrm{Jul}}^{\mathrm{W}}\left(U_{\mathrm{Jul}}^{S}\right)$ and $U_{\mathrm{Oct}}^{\mathrm{W}}\left(U_{\mathrm{Oct}}^{S}\right)$, respectively. We regard these El Niño events as "observed El Niño events" to be predicted and then compute their CNOP, CNOP-I and CNOP-P errors, finally determining the dominant source of the errors that yield considerable prediction uncertainties.

Using the uncertain initial condition and model parameters, we predict the above predetermined El Niño events with a one year lead time and the respective initial precursory time as the start time of predictions. In the WF96 model, we consider the initial errors of $T$ and $h$, denoted by $u_{0}=\left(T_{0}^{\prime}, h_{0}^{\prime}\right)$; and the uncertainties of the parameters $P=(\alpha, \mu)$, signified by $p^{\prime}=\left(\alpha^{\prime}, \mu^{\prime}\right)$. In this case, $U_{0}$ and $u_{0}$ in the objective function (2.3) related to CNOPs represents the initial state $\left(T_{0}, h_{0}\right)$ of the model El Niño events and its initial error $\left(T_{0}^{\prime}, h_{0}^{\prime}\right), P$ and $p^{\prime}$ stand for the parameters $\alpha$ and $\mu$ and their errors $\alpha^{\prime}$ and $\mu^{\prime}$, and the objective function $J=\sqrt{T^{\prime}(\tau)^{2}+h^{\prime}(\tau)^{2}}$ measures the resultant prediction errors by $\left(T_{0}^{\prime}, h_{0}^{\prime}\right)$ and $\left(\alpha^{\prime}, \mu^{\prime}\right)$ at the prediction time $\tau$, respectively. 
The norm $\left\|u_{0}\right\|=\max \left\{\left|T_{0}^{\prime}\right|,\left|h_{0}^{\prime}\right|\right\}$ is used to measure the magnitudes of initial errors. And the constraint

$C_{\delta}=\left\{u_{0} \mid\left\|u_{0}\right\| \leq \delta\right\}$

with $\delta$ ranging from 0.01 to 0.05 is adopted to control the magnitudes of initial errors. For parameter errors $\alpha^{\prime}$ and $\mu^{\prime}$, we determine their constraints according to the basic rules of numerical simulation of ENSO. In realistic ENSO predictions, the forecast model should first guarantee that it can simulate the main features of the observed ENSO. As such, the values of the parameters in the model must be set to satisfy this precondition. Keep this in mind, as we determine that the parameter errors satisfy the constraint

$C_{\sigma}=\left\{\left(\alpha^{\prime}, \mu^{\prime}\right)|| \alpha^{\prime}\left|/ \alpha \leq \sigma_{1},\right| \mu^{\prime} \mid / \mu \leq \sigma_{2}\right\}$

with $\sigma_{1}=0.03$ and $\sigma_{2}=0.08$.

With the constraint $C_{\delta}$, Mu and Duan (2003) computed the CNOP-I superimposed on some El Niño events in the WF96 model and demonstrated that the CNOP-I errors always have the patterns of $(-\delta, \delta)$. In this paper, we obtain the CNOP-I of the predetermined eight El Niño events, which are similar to those of $\mathrm{Mu}$ and Duan (2003) and have the common patterns $(-\delta, \delta)$ with $\delta$ ranging from 0.01 to 0.05 . In the perfect model scenario, these CNOP-I errors have the largest effect on prediction uncertainties of the corresponding El Niño events for the constraint $C_{\delta}$ and make the corresponding El Niño events to be over-predicted.

In order to explore the dominant source of the errors that yield considerable prediction uncertainties for ENSO events, we also include the effect of parameter errors in the model and investigate the CNOP and CNOP-P errors of the El Niño events.

To compute the CNOP and CNOP-P, we need the information of the gradient of the objective function $J$, similar to the computation of CNOP-I. According to Eq. (3.3) and the Eqs. (3.6)-(3.8), we construct the adjoint model of the ENSO model (4.1) and obtain the gradient of the objective function $J$ with respect to the initial perturbations and the parameter perturbations. By using this gradient information, the CNOP and CNOP-P errors of the eight El Niño events are computed by the L-BFGS-B solver (Liu and Nocedal, 1989; Zhu et al., 1997), the algorithm which was also used to obtain CNOP-I in Mu and Duan (2003) and Duan et al. (2009).

The CNOP errors of the predetermined eight El Niño events are first investigated. The results demonstrate that there exists one CNOP error for each El Niño prediction for each of different magnitudes of constraints; furthermore, the CNOP errors of different El Niño predictions always correspond to initial perturbations that lie on the boundary of the domain defined by the constraint and have similar patterns. For simplicity, we only list the ones, in Table 1, of the predictions of the El Niño event $U_{\text {Jan }}^{\mathrm{W}}$ for different constraints. From the definition of CNOP, it is known that the CNOP errors cause the largest prediction errors for the combined modes
Table 1. CNOPs for the reference state El Niño event $U_{\text {Jan }}^{\mathrm{W}}$

\begin{tabular}{lllll}
\hline$C_{\delta}$ and $C_{\sigma}$ & $T_{0}^{\prime}$ & $h_{0}^{\prime}$ & $\alpha^{\prime}$ & $\mu^{\prime}$ \\
\hline$\delta=0.01, \sigma_{1}=0.03, \sigma_{2}=0.08$ & -0.01 & 0.01 & 0.0006 & -0.1220 \\
$\delta=0.02, \sigma_{1}=0.03, \sigma_{2}=0.08$ & -0.02 & 0.02 & 0.0006 & -0.1220 \\
$\delta=0.03, \sigma_{1}=0.03, \sigma_{2}=0.08$ & -0.03 & 0.03 & 0.0006 & -0.1220 \\
$\delta=0.04, \sigma_{1}=0.03, \sigma_{2}=0.08$ & -0.04 & 0.04 & 0.0006 & -0.1220 \\
$\delta=0.05, \sigma_{1}=0.03, \sigma_{2}=0.08$ & -0.05 & 0.05 & 0.0006 & -0.1220 \\
\hline
\end{tabular}

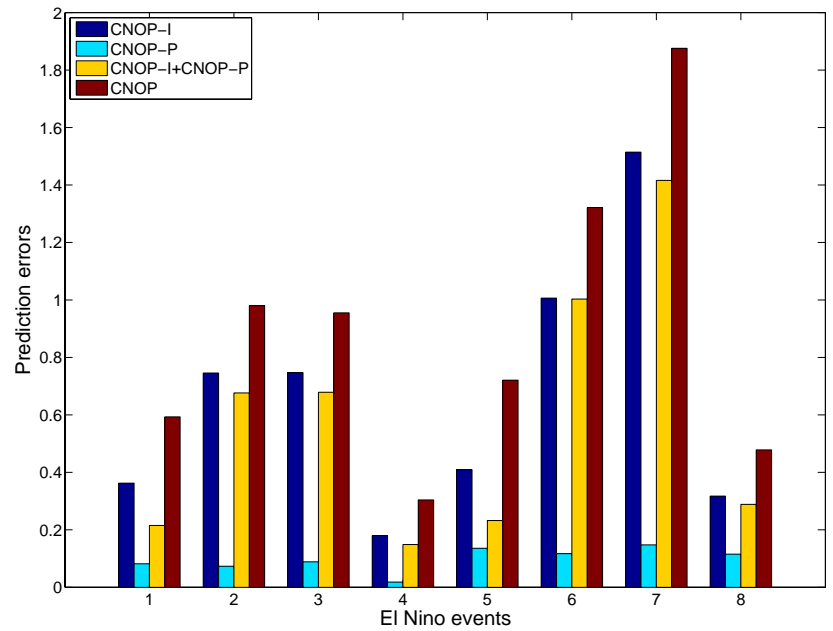

Fig. 1. Magnitudes of prediction errors caused by the CNOPI, CNOP-P, CNOP errors, and the combination of CNOP-I and CNOP-P errors with $\delta=0.05$ and $\sigma_{1}=0.03$ and $\sigma_{2}=0.08$, for the predetermined eight model El Niño events. The measurement of the prediction errors is $J\left(u_{0} ; p^{\prime}\right)=\sqrt{T^{\prime}(\tau)^{2}+h^{\prime}(\tau)^{2}}$. The numbers 1 , 2,3 and 4 in the horizontal axis denote the El Niño events $U_{J a n}^{\mathrm{W}}$, $U_{\mathrm{Apr}}^{\mathrm{W}}, U_{\mathrm{Jul}}^{\mathrm{W}}$ and $U_{\mathrm{Oct}}^{\mathrm{W}}$; and 5, 6, 7 and 8 signify the El Niño events $U_{\mathrm{Jan}}^{\mathrm{S}}, U_{\mathrm{Apr}}^{\mathrm{S}}, U_{\mathrm{Jul}}^{\mathrm{S}}$ and $U_{\mathrm{Oct}}^{\mathrm{S}}$, respectively.

of initial errors and parameter errors in a constraint. To illustrate them, we plot, in Fig. 1, the prediction errors caused by CNOP errors with $\delta=0.05$. By comparing the prediction errors caused by CNOP errors and CNOP-I errors, we find that the CNOP errors only lead to a slightly larger prediction error than the CNOP-I errors. This indicates that, even if the maximum allowable parameter errors are considered in the WF96 model, they have, at most, trivial effects on the prediction errors caused by initial errors. It is implied that initial errors may be the dominant source of the errors that cause notable uncertainties of ENSO predictions.

Now we study the CNOP-P errors for the eight El Niño events. In this case, we assume initial states of reference state El Niño events are perfect, then only consider the effect of parameter errors on ENSO predictability. The optimization problem (2.6) in Sect. 2 is related to CNOP-P, where $U_{0}$, $P$ and $p^{\prime}$ are the same as those of CNOP, but the objective function $J=\sqrt{T^{\prime}(\tau)^{2}+h^{\prime}(\tau)^{2}}$ only measures the prediction 
error of the El Niño events caused by the parameter errors $\left(\alpha^{\prime}, \mu^{\prime}\right)$ in the constraint $C_{\sigma}$ in (4.3). With the gradient of $J$, with respect to $\left(\alpha^{\prime}, \mu^{\prime}\right)$, the CNOP-P for the eight El Niño events are calculated. The results show that, for the constraint $C_{\sigma}$ with $\sigma_{1}=0.03$ and $\sigma_{2}=0.08$, the CNOP-P errors for eight El Niño events are coherently of the pattern $(0.03 \alpha, 0.08 \mu)$ with $\alpha=0.0212$ and $\mu=1.525$ and correspond to the parameter perturbations that lie on the boundary of the domain defined by the constraint $C_{\sigma}$. Furthermore, we find that these CNOP-P errors are not the same as the parameter perturbation component in the CNOP patterns obtained above. We note that the CNOP-P errors cause the corresponding El Niño events to be under-predicted; while the parameter error component $(0.03 \alpha,-0.08 \mu)$ in the CNOP errors cause the El Niño events to be over-predicted (figures are omitted). This indicates that CNOP in the WF96 model could not be a simple combination of CNOP-I and CNOP-P, which then may imply that CNOP errors consider the effect of nonlinear interaction between initial errors and model parameter errors. In addition, the CNOP-I errors, as described above, cause the model El Niño events to be over-predicted; furthermore, the initial error component in the CNOP errors is the same as the CNOP-I errors and also cause the corresponding El Niño events to be over-predicted. It is conceivable that, if the model parameter error components in the CNOP errors are the same as the CNOP-P patterns, they will offset the prediction errors caused by the initial error components of the CNOP errors. This fact illustrates physically why the CNOP error, rather than the combination of CNOP-I and CNOP-P, is the optimal one in the WF96 model and has the largest effect on prediction uncertainties for El Niño events. Furthermore, we note that the differences between the prediction errors caused by the CNOP errors and those caused by the combination of CNOP-I and CNOP-P errors reflect the effect of nonlinearity, due to the nonlinear response of initial errors to the parameter errors. Of course, the nonlinearities shown in CNOP errors, similar to those in CNOP-I errors (see $\mathrm{Mu}$ et al., 2007a), can also be extracted from the difference between the prediction errors in nonlinear model and those in its corresponding linearized one. Therefore, although we have demonstrated that the model parameter errors play a minor role in yielding significant uncertainties of ENSO predictions in the WF96 model, the differences among CNOP, CNOP-I and CNOP-P errors suggest that, to better estimate the predictability limit and reveal the nonlinear effect, the optimal combined mode of initial error and model parameter errors should be investigated.

\section{Summary and discussion}

This study presents an extension of the CNOP approach, which has renewed the CNOP as the optimal combined mode of initial perturbation and parameter perturbation, i.e. the one that causes the largest departure from the reference state. The renewed CNOP has two special cases: one is CNOP-I that is only related to initial perturbation and induces the largest nonlinear evolution at prediction time; the other is CNOP$\mathrm{P}$, which is proposed in this paper and merely associated with the parameter perturbation, causing the largest departure from the reference state.

CNOP-I acts as the initial error that has the largest effect on prediction uncertainties in a perfect model scenario; while the CNOP can represent the optimal combined mode of initial errors and model parameter errors, which causes the maximum prediction error; and CNOP-P can stand for the parameter error that yields the maximum prediction errors in perfect initial condition scenario. This physics of CNOPs allows us to investigate, not only the first kind of predictability problems but also the second kind of ones; furthermore, the predictability problems of the coexistence of initial errors and model parameter errors. In this paper, we use such physics of the CNOPs to study the ENSO predictability by a theoretical coupled ocean-atmosphere model. It is demonstrated that the CNOP errors only cause a slightly larger prediction error than the CNOP-I errors. This indicates that initial errors, rather than model parameter errors, may play the major role in yielding notable prediction uncertainties for ENSO events. It is clear that the CNOP errors consider the combined effect of initial errors and model parameter errors and enable itself to investigate the relative effect of initial errors and model parameter errors on prediction uncertainties and to identify the dominant source of the uncertainties that yield a big effect on predictability. In addition, we show that the CNOP errors are not a simple combination of CNOP-I and CNOP-P errors, but a particular combined pattern of initial errors and model parameter errors. Furthermore, this particular error pattern and its resultant prediction error consider sufficiently an effect of nonlinearity. Therefore, to better estimate the predictability limit and reveal nonlinear effect, we should explore the CNOP errors, i.e. the optimal combined mode of initial errors and model parameter errors.

CNOP-I also manifested its physics as the optimal precursor of a weather or a climate event, and the most sensitive (or most unstable) initial pattern in sensitivity analysis (Duan et al., 2004; Mu et al., 2004). It is expected that CNOP-P could illuminate the most sensitive parameter perturbation, and provide information on determining the sequence of sensitivity of model parameters. It is known that there are many parameters in numerical models and most of these parameters are determined by observations. The CNOP-P may help determine the parameters that should be better fixed by observations. For the CNOP, it could be used to find the optimal precursor of a weather or a climate event under the optimal parameter condition, in an attempt to study the effect of model parameters' sensitivity on the precursors. CNOP may also be applied to investigate the most unstable (sensitive) initial modes with the most sensitive parameter perturbations in sensitivity analysis. Of course, these physics of CNOP should be realized by applying them to physical problems of 
interest. Nevertheless, what should be pointed out is that the CNOP-P here is only related to model parameter errors and cannot consider other kinds of model errors. Despite this, it is expected that CNOP will play an important role in the studies of atmospheric and oceanic sciences.

Acknowledgements. We thank the anonymous reviewer very much for his useful comments. We also appreciate Olivier Talagrand for his note on the derivation of the gradient formula, for his correcting errors in the first version of this paper and for his very insightful comments. This work was jointly sponsored by Knowledge Innovation Program of the Chinese Academy of Sciences (No. KZCX2-YW-QN203), the National Basic Research Program of China (Nos. 2006CB403606; 2007CB411800), the National Natural Science Foundation of China (No. 40830955).

Edited by: O. Talagrand

Reviewed by: two anonymous referees

\section{References}

Aanonsen, S. I.: Efficient history matching using a multiscale technique, SPE Reservoir Evaluation and Engineering, 154-164, February 2008.

Birgin, E. G., Martinez, J. M., and Raydan, M.: Nonmonotone spectral projected gradient methods on convex sets, SIAM J. Optimiz., 10, 1196-1211, 2000.

Chu, P. C., Lu, S. H., and Chen, Y. C.: Two kinds of predictability problems in Lorenz system and climate models, Second Hayes Symposium on Seasonal to Interannual Climate Variability-The 1997/1998 ENSO Cycle, American Meteorological Society, 93$100,1999$.

Cai, M., Kalnay, E., and Toth, Z.: Bred vectors of the Zebiak-Cane model and their potential application to ENSO predictions, J. Climate, 16, 40-55, 2003.

Duan, W. S., Mu, M., and Wang, B.: Conditional nonlinear optimal perturbation as the optimal precursors for El NiñoSouthern Oscillation events, J. Geophys. Res., 109, D23105, doi:10.1029/2004JD004756, 2004.

Duan, W. S. and Mu, M.: Investigating decadal variability of El Niño-Southern Oscillation asymmetry by conditional nonlinear optimal perturbation, J. Geophys. Res., 111, C07015, doi:10.1029/2005JC003458, 2006.

Duan, W. S., Xu, H., and Mu, M.: Decisive role of nonlinear temperature advection in El Niño and La Niña amplitude asymmetry, J. Geophys. Res., 113, C01014, DOI:10.1029/2006JC003974, 2008.

Duan, W. S., Liu, X. C., Zhu, K. Y., and Mu, M.: Exploring the initial errors that cause a significant "spring predictability barrier" for El Niño events, J. Geophys. Res., 114, C04022, doi:10.1029/2008JC004925, 2009.

Duan, W. S. and Mu, M.: Conditional nonlinear optimal perturbation: applications to stability, sensitivity, and predictability, Sci. China Ser. D, 52(7), 883-906, 2009.

Duan, W. S., Xue, F., and Mu, M.: Investigating a nonlinear characteristic of El Niño events by conditional nonlinear optimal perturbation, Atmos. Res., 94, 10-18, 2009.

Fan, X. G. and Chou, J. F.: Methods and Experiments of Numerical Prediction Raised as Inverse Problem Part I: Three Kinds of In- verse Problems and Numerical Solutions, Chinese J. Atmos. Sci., 23, 543-550, 1999 (in Chinese).

Grimstad, A., Mannseth, T., Nevdal, G., and Urkedal, H.: Adaptive multiscale permeability estimation, Comput. Geosci., 7, 1-25, 2003.

Houtekamer, P. L. and Derome, J.: Methods for ensemble prediction, Mon. Weather Rev., 123, 2181-2196, 1995.

Hamill, T. M., Snyder, C., and Morss, R. E.: A comparison of probabilistic forecasts from bred, singular-vector, and perturbed observation en-sembles, Mon. Weather Rev., 128, 1835-1851, 2000.

Jin, E. K., Kinter III, J. L., Wang, B., et al.: Current status of ENSO prediction skill in coupled ocean-atmosphere models, Clim. Dynam., 31, 647-664, 2008.

Le Dimet, F. X. and Talagrand, O.: Variational algorithms for analysis and assimilation of meteorological observations: Theoretical aspects, Tellus A, 38, 97-110, 1986.

Lorenz, E.: A study of the predictability of a 28-variable atmospheric model, Tellus, 17, 321-333, 1965.

Lorenz, E. N.: Climate predictability: The physical basis of climate modelling, Global Atmospheric Research Programme Publication Series, No. 16, World Meteorology Organization Geneva, 132-136, 1975.

Liu, D. C. and Nocedal, J.: On the Limited Memory Method for Large Scale Optimization, Math. Program., 45(3), 503-528, 1989.

Liu, Z. Y.: A simple model study of ENSO suppression by external periodic forcing, J. Atmos. Sci., 15, 1088-1098, 2002.

$\mathrm{Mu}, \mathrm{M}$ : : Nonlinear singular vectors and nonlinear singular values, Sci. China Ser. D, 43, 375-385, 2000.

Lu, J. and Hsieh, W. W.: On determining initial conditions and parameters in a simple coupled atmosphere-ocean model by adjoint data assimilation, Tellus A, 50, 534-544, 1998.

Moore, A. M. and Kleeman, R.: The dynamics of error growth and predictability in a coupled model of ENSO, Q. J. Roy. Meteor. Soc., 122, 1405-1446, 1996.

Mu, M., Duan, W. S., and Wang, J. C.: Predictabili problems in numerical weather and climate prediction, Adv. Atmos. Sci., 19, 191-205, 2002.

Mu, M. and Zhang, Z. Y.: Conditional nonlinear optimal perturbation of a Two-Dimensional Quasigeostrophic Model, J. Atmos. Sci., 63, 1587-1604, 2006.

$\mathrm{Mu}, \mathrm{M}$. and Duan, W. S.: A new approach to studying ENSO predictability: conditional nonlinear optimal perturbation, Chinese Sci. Bull., 48, 1045-1047, 2003.

Mu, M., Mu, M., Duan, W. S., and Wang, B.: Conditional nonlinear optimal perturbation and its applications, Nonlin. Processes Geophys., 10, 493-501, 2003, http://www.nonlin-processes-geophys.net/10/493/2003/.

$\mathrm{Mu}$, M., Sun, L., and Dijkstra, H.: The sensitivity and stability of the ocean's thermocline circulation to finite amplitude freshwater perturbations, J. Phys. Oceanogr., 34, 2305-2315, 2004.

Mu, M., Duan, W. S., and Wang, B.: Season-dependent dynamics of nonlinear optimal error growth and El Niño-Southern Oscillation predictability in a theoretical model, J. Geophys. Res., 112, D10113, doi:10.1029/2005JD006981, 2007a.

Mu, M., Xu, H., and Duan, W.: A kind of initial errors related to "spring predictability barrier" for El Niño events in Zebiak-Cane model, Geophys. Res. Lett., 34, L03709, 
doi:10.1029/2006GL027412, 2007b.

Mu, M. and Jiang, Z. N.: A Method to find out the perturbations triggering the blocking onset: Conditional nonlinear optimal perturbations, J. Atmos. Sci., 65, 3935-3946, 2009.

Mu, M., Zhou, F., and Wang, H.: A Method for Identifying the Sensitive Areas in Targeted Observations for Tropical Cyclone Prediction: Conditional Nonlinear Optimal Perturbation, Mon. Weather Rev., 137, 1623-1639, 2009.

Sun, L., Mu, M., Sun, D. J., and Yin, X. Y.: Passive mechanism decadal variation of thermohaline circulation, J. Geophys. Res., 110, C07025, doi:10.1029/2005JC002897, 2005.

Orrell, D.: Forecast error growth, imperfect models, and climatology of the two-scale Lorenz system, Transfer of status thesis, University of Oxford, Oxford, 1999.

Orrell, D.: Model error and predictability over different timescales in the Lorenz'96 systems, J. Atmos. Sci., 60, 2219-2228, 2003.

Powell, M. J. D.: VMCWD: A FORTRAN subroutine for constrained optimization. DAMTP Report 1982/NA4, University of Cambridge, England, 1982.

Rosen, S. A.: The gradient projection method for nonlinear programming. Part I: Linear constraints, J. Soc. Ind. Appl. Math., 8(1), 181-217, 1960.

Riviere, O., Lapeyre, G., and Talagrand, O.: Nonlinear generalization of singular vectors: Behavior in a baroclinic unstable flow, J. Atmos. Sci., 65, 1896-1911, 2008.

Riviere, O., Lapeyre, G., and Talagrand, O.: A novel technique for nonlinear sensitivity analysis: application to moist predictability, Q. J. Roy. Meteor. Soc., 135, 1520-1537, 2009.

Samelson, R. G. and Tziperman, E.: Instability of the chaotic ENSO: The growth-phase predictability barrier, J. Atmos. Sci., 58, 3613-3625, 2001.

Shutyaev, V., Le Dimet, F.-X., and Gejadze, I.: On optimal solution error covariances in variational data assimilation, Russ. J. Numer. Anal. M., 23, 185-206, 2008.
Tang, Y., Deng, Z., Zhou, X., Cheng, Y., and Chen, D.: Interdecadal variation of ENSO predictability in multiple models, J. Climate, 21, 4811-4833, 2008.

Terwisscha van Scheltinga, A. D. and Dijkstra, H. A.: Conditional nonlinear optimal perturbations of the double-gyre ocean circulation, Nonlin. Processes Geophys., 15, 727-734, 2008, http://www.nonlin-processes-geophys.net/15/727/2008/.

Toth, Z. and Kalnay, E.: Ensemble forecasting at NCEP and the breeding method, Mon. Weather Rev., 127, 3297-3318, 1997.

Thompson, C. J.: Initial conditions for optimal growth in a coupled ocean-atmosphere model of ENSO, J. Atmos. Sci., 55, 537-557, 1998.

Wang, B. and Fang, Z.: Chaotic oscillation of tropical climate: A dynamic system theory for ENSO, J. Atmos. Sci., 53, 27862802, 1996.

Wang, C. and Picaut, J.: Understanding ENSO physics - A review, in: Earth Climate: The Ocean-Atmosphere Interaction, American Geophysical Union, Geoph. Monog. Series, 147, 21-48, 2004.

William, P. D.: Modelling climate change: the role of unresolved processes, Philos. T. R. Soc. A, 363, 2931-2946, 2005.

Wu, D. H. Anderson, D. L.: ENSO variability and external impacts, J. Climate, 6, 1703-1717, 1993.

Wu, X. G. and Mu, M.: Impact of horizontal diffusion on the nonlinear stability of thermohaline circulation in a modified box model, J. Phys. Oceanogr., 39, 798-805, 2009.

Xue, Y., Cane, M. A., and Zebaik, S. E.: Predictability of a coupled model of ENSO using singular vector analysis. Part I: Optimal growth in seasonal background and ENSO cycles, Mon. Weather Rev., 125, 2043-2056, 1997.

Zebiak, S. E. and Cane, A.: A model El Niño-Southern oscillation, Mon. Weather Rev., 115, 2262-2278, 1987.

Zhu, C., Byrd, R. H., and Nocedal, J.: L-BFGS-B: Algorithm 778: L-BFGS-B, FORTRAN routines for large scale bound constrained optimization, ACM T. Math. Software, 23(4), 550-560, 1997. 